\title{
Experimental study of low-frequency electroacupuncture-induced differentiation of bone marrow mesenchymal stem cells into chondrocytes
}

\author{
GUANGWEN WU ${ }^{1}$, JUN PENG ${ }^{1}$, MINGXIA WU ${ }^{1}$, YURONG LI $^{2}$, YUNMEI HUANG ${ }^{1}$, \\ RUHUI LIN ${ }^{1}$, QIAOYAN CAI ${ }^{1}$ and XIANXIANG LIU ${ }^{1}$ \\ ${ }^{1}$ Academy of Integrative Medicine, Fujian University of Traditional Chinese Medicine, Fuzhou 350108; \\ ${ }^{2}$ Institute of Precision Instruments, Fuzhou University, Fuzhou 350002, P.R. China
}

Received August 16, 2010; Accepted October 5, 2010

DOI: $10.3892 / \mathrm{ijmm} .2010 .555$

\begin{abstract}
In the present study, we investigated the effect of low-frequency electroacupuncture (EA) on the differentiation of bone mesenchymal stem cells (BMSCs) into chondrocytes and the molecular mechanism involved. We isolated BMSCs from Sprague-Dawley (SD) rat bone marrow. Third-generation SD rat BMSCs (P3 BMSCs) were harvested and characterized by flow cytometry with FITC staining. Data indicated that the positive rates of CD90 and CD45 were 98.22 and $1.91 \%$, respectively, indicating the high purity of the BMSCs. The P3 BMSCs were treated with EA for 15 or 30 min daily for 7 or 14 days. Using optical microscopy and transmission electron microscopy, we found that EA induced morphological changes in the BMSCs, displaying typical morphology of early chondrocytes. In addition, we found that the cytoplasm and extracellular matrices were metachromatically stained by toluidine blue in the treated cells in a dose-dependent manner, indicating that EA treatment resulted in the expression of glycosaminoglycan. Furthermore, upon immunohistocytochemical staining and Western blotting, we found that EA treatment significantly and dose-dependently induced expression of chondrocyte-specific matrix protein type II collagen, which may have been mediated by the transcription factor Sox9, as the mRNA expression of Sox9 was found to be significantly increased after EA treatment. Taken together, these results suggest that EA can be employed as a novel non-drug-inducing method for the differentiation of BMSCs into chondrocytes.
\end{abstract}

Correspondence to: Dr Xianxiang Liu, Academy of Integrative Medicine, Fujian University of Traditional Chinese Medicine, 1 Huatuo Road, Minhou Shangjie, Fuzhou 350108, P.R. China E-mail: liuxianxiang@163.com

Key words: bone mesenchymal stem cells, low-frequency electroacupuncture, differentiation, chondrocytes, tissue engineering

\section{Introduction}

Bone marrow mesenchymal stem cells (BMSCs) are multipotent stem cells derived from the mesoderm, which are capable of transdifferentiation and differentiation into osteoblasts, chondrocytes, adipocytes, myoblasts and neural cells, and are the favored seed cells for repairing joint and cartilage defects and for other tissue engineering applications (1-3). It has been documented that BMSCs are characterized by several phenotypes, including antigens such as $\mathrm{CD} 90^{+}, \mathrm{SH} 2^{+}$, $\mathrm{SH}^{+}, \mathrm{CD}_{4} 4^{+}, \mathrm{CD}_{29}{ }^{+}, \mathrm{CD}_{11}{ }^{+}, \mathrm{CD}_{106}{ }^{+}, \mathrm{CD}_{120 \mathrm{a}^{+}}$and $\mathrm{CD} 124^{+}$, but not by other hemopoietic surface markers, such as CD45, CD11b, CD14, CD34 and CD31 (4). Thus, the purity of BMSCs can be preliminarily measured by flow cytometry or immunocytochemical analysis with positive staining of CD90 and CD45 (5).

Methods which effectively induce the committed differentiation of stem cells has become the focus of tissue engineering development. An effective and safe modality of induction is valuable since potential safety concerns have arisen as a result of the previous differentiation of BMSCs induced by agents $(6,7)$.

Acupuncture, which has been used for the treatment of various types of diseases in Eastern countries for thousands of years, is currently gaining acceptance as an alternative medicine in Western countries $(8,9)$. Electroacupuncture (EA) is a modified acupuncture technique that utilizes electrical stimulation. Previous studies have demonstrated that EA has therapeutic effects on chondral defects including knee osteoarthritis (10-12) and produces cytokines with multiple biological activities in various types of diseases (13-15). In addition, animal experiments have shown that EA enhances BMSC differentiation into neuronal cells (16). However, the effects of EA on the differentiation of BMSCs into chondrocytes, have not yet been reported.

To seek an effective and safe way to induce the differentiation of BMSCs into chondrocytes and justify the efficacy of EA in the treatment of chondral defects, in the present study, we isolated and purified BMSCs from Sprague-Dawley (SD) rats and evaluated the effects of EA on the differentiation of BMSCs into chondrocytes, and investigated its underlying molecular mechanism. 
A1

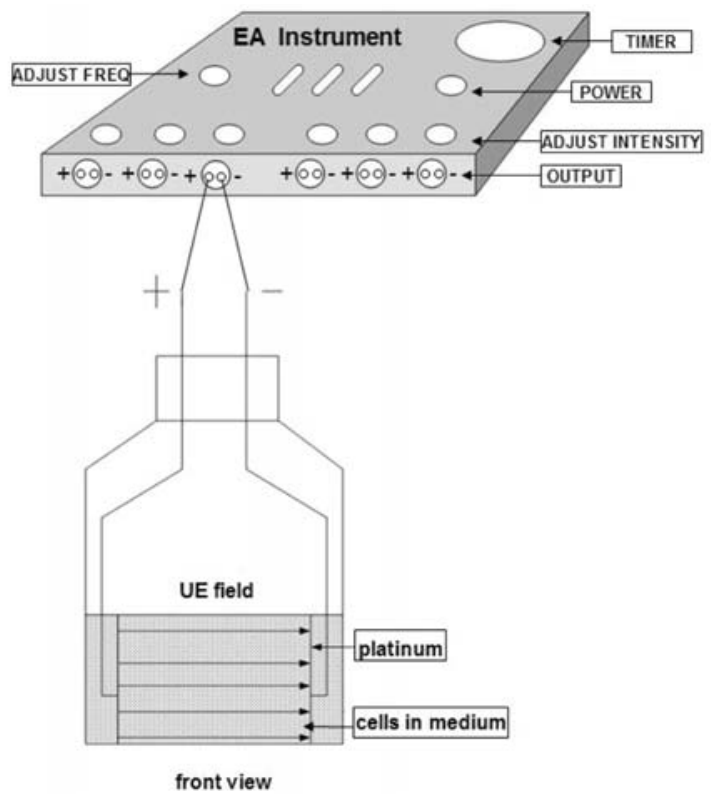

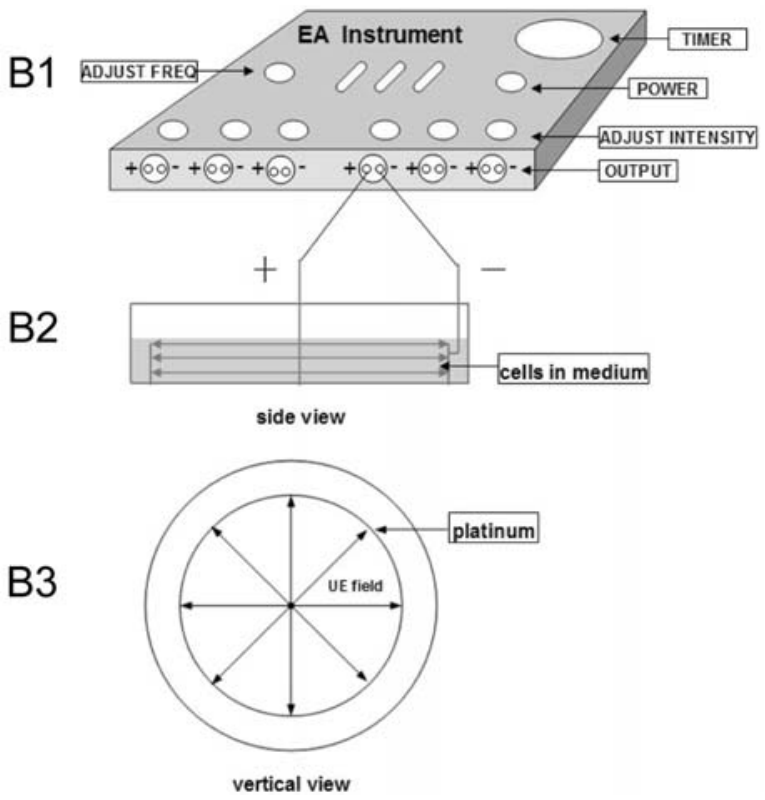

Figure 1. EA treatment method. P3 BMSCs cultured in a culture flask were treated as illustrated. A1 (EA instrument, stereogram), A2 (front view), B1 (EA instrument, stereogram), B2 (side view) and B3 (vertical view) in a 3.5-cm Petri dish. The linking lines set in the culture liquid were platinum, which are non-erosive.

\section{Materials and methods}

Reagents. Fetal bovine serum (FBS), Dulbecco's modified Eagle's medium (DMEM) and trypsin were purchased from Hyclone Laboratories, Inc. (Logan, UT, USA). Toluidine blue stain and platinum were obtained from Sinopharm Chemical Reagent Co. Ltd. (Shanghai, China). Anti-CD45FITC, anti-CD90-FITC and anti-mouse IgG-FITC were purchased from Santa Cruz Biotechnology, Inc. (Santa Cruz, CA, USA). The mammalian cell lysis buffer was obtained from Thermo Scientific Pierce Protein Research Products (M-PER; Rockford, IL, USA). The protease inhibitor cocktail was purchased from EMD Biosciences Inc. (San Diego, CA, USA). The phosphatase inhibitor cocktail was purchased from Sigma Chemical Co. (St. Louis, MO, USA). Rabbit anti-rat type II collagen and HRP secondary goat anti-rabbit antibodies were purchased from EMD Chemicals Inc. (Darmstadt, Germany). The Chemiluminescent Western blot immunodetection kit was obtained from Invitrogen Inc. (Grand Island, NY, USA). The antibody against B-actin was obtained from Cell Signaling Technology, Inc. (Danvers, MA, USA). DNA primers were synthesized by Sangon Biotech (Shanghai, China). Rabbit anti-rat type II collagen antibody, the Ultrasensitive ${ }^{\mathrm{TM}} \mathrm{S}-\mathrm{P}$ and DAB kits were purchased from Zhongshan Goldenbridge Biotech (Beijing, China).

Animals. Healthy and clean SD rats of either gender $(n=45)$, aged 4 weeks and weighing 90-110 g, were purchased from SLAC Laboratory Animal Inc., Shanghai, China [Laboratory animal use certificate no. SCXK(SH)2007-0005] and raised in a sterile environment. The care and use of the laboratory animals complied with the Guidance Suggestions for the Care and Use of Laboratory Animals (2006) advocated by the Ministry of Science and Technology, China (17).
Isolation, purification and culture of BMSCs. SD rats were sacrificed using cervical dislocation, and the bilateral femora and tibiae were sterilely retrieved. Bones were rinsed with $75 \%$ ethanol for 3-5 min, and marrow cavities were rinsed with DMEM containing 15\% FBS. Cells were cultured in 25-ml culture flasks at a density of $1 \times 10^{5}$ cells $/ \mathrm{ml}$ in $5 \mathrm{ml}$ DMEM containing $15 \% \mathrm{FBS}$ at $37^{\circ} \mathrm{C}$ and $5 \% \mathrm{CO}_{2}$ (named $\mathrm{P} 0$ ). The culture media were refreshed $72 \mathrm{~h}$ later and subsequently at an interval of 3 days. The cells were subcultured at $80-90 \%$ confluency, sequentially named P1, P2 and P3. Nucleated cells were counted with a hemocytometer, and the cell density was adjusted for further experiments.

Detection of surface antigens CD45 and CD90 in P3 BMSCs. The P3 BMSCs were harvested and incubated with saturated CD45-FITC and CD90-FITC monoclonal antibodies at room temperature in the dark for $25 \mathrm{~min}$ and were analyzed by flow cytometry (Becton Dickinson, Franklin Lakes, NJ, USA). Mouse IgG-FITC was used as an isotype control.

EA stimulation. As shown in Fig. 1, a pair of platinum links was set in $25-\mathrm{ml}$ culture flasks or $3.5-\mathrm{cm}$ Petri dishes. As one of them was sufficiently wide and long, based on the Gauss theorem, we concluded that the culture solution was affected by a uniform electric (UE) field, neglecting the edge. Therefore, the cells were able to receive a homogeneous effect. The platinum links were then connected to an electrical stimulator (Suzhou Medical Appliance Factory Co., Ltd., China) and stimulated electrically with low-frequency EA of $2 \mathrm{~Hz}$ with square-wave burst pulses (duration $1.1 \mathrm{sec}$ ) and a burst frequency of $100 \mathrm{~Hz}$ (duration $2.2 \mathrm{sec}$ ) with alternating polarity. The intensity of the output voltage was $17.3 \mathrm{~V}$ and the pulse width was shorter than $1 \mathrm{msec}$. The acupuncture stimulation was applied for 15 or $30 \mathrm{~min}$, daily. 
Cell treatment. P3 BMSCs were digested with $0.25 \%$ trypsin and incubated in $25-\mathrm{ml}$ culture flasks at a density of $1 \times 10^{5}$ cells/ $\mathrm{ml}$ in $5 \mathrm{ml}$ medium and in $3.5-\mathrm{cm}$ Petri dishes at a density of $1 \times 10^{5}$ cells $/ \mathrm{ml}$ in $2 \mathrm{ml}$ medium for $24 \mathrm{~h}$. Cells subsequently received EA treatment daily for 15 or $30 \mathrm{~min}$ daily for 7 or 14 days.

Observation of morphological changes. P3 BMSCs were seeded into $25-\mathrm{ml}$ culture flasks at a density of $1 \times 10^{5}$ cells $/ \mathrm{ml}$ in $5 \mathrm{ml}$ medium. The cells were treated with EA for 15 or 30 min daily for 7 or 14 days. Cell morphology was observed using a phase-contrast microscope (Olympus, Japan) and transmission electron microscopy (TEM) (Hitachi, Ltd., Japan). Images were captured at a magnification of x100 for phase-contrast microscopy or x13,000 or x18,000 for TEM.

Toluidine blue staining and analysis of positivity. P3 BMSCs $\left(2 \times 10^{5}\right)$ were seeded into $3.5-\mathrm{cm}$ Petri dishes set with a coverslip in $2 \mathrm{ml}$ medium and treated with EA for 15 or $30 \mathrm{~min}$ daily for 14 days. The expression of glycosaminoglycan was observed using toluidine blue staining. Images were captured at a magnification of $\times 100$, and the positivity was analyzed using the Motic Med 6.0 Digital Medical imaging analysis system.

Immunohistocytochemical staining and analysis of positivity. P3 BMSCs $\left(2 \times 10^{5}\right)$ were seeded into $3.5-\mathrm{cm}$ Petri dishes set with a coverslip in $2 \mathrm{ml}$ medium and treated with EA for 15 or 30 min daily for 14 days. The expression of type II collagen was observed using immunocytochemical staining. Images were captured at a magnification of $\mathrm{x} 100$, and the positivity was analyzed using the Motic Med 6.0 Digital Medical imaging analysis system.

Western blot analysis. P3 BMSCs were seeded into 25-ml culture flasks at a density of $1 \times 10^{5}$ cells $/ \mathrm{ml}$ in $5 \mathrm{ml}$ medium. The cells were treated with EA for 15 or 30 min for 14 days. The treated cells were lysed with mammalian cell lysis buffer containing protease and phosphatase inhibitor cocktails, and the lysates were resolved in 12\% SDS-PAGE gel and electroblotted using the iBlot Western detection stack/iBlot dry blotting system (Invitrogen). The PVDF membranes were blocked with Blocking Solution for $1 \mathrm{~h}$ and rinsed with water three times, 5 min each time, followed by incubation with Primary Antibody Solution (1:4,000) for $60 \mathrm{~min}$ and rinsed with Prepared Antibody wash three times, 5 min each time. The membranes were then incubated with Secondary Antibody Solution $(1: 2,500)$ for $30 \mathrm{~min}$ and rinsed with prepared Antibody Wash three times, 5 min each time. Finally, the antibody-bound protein bands were detected with ECL, and images were captured using the P-14 automatic processor (Suzhou Proud Precision Machinery Co., Ltd., China). Using B-actin as an internal control, the grayscale value ratio of the target protein to the internal control was used to measure the relative amount of collagen type II.

RNA extraction and RT-PCR analysis. P3 BMSCs were seeded into $25-\mathrm{ml}$ culture flasks at a density of $1 \times 10^{5}$ cells $/ \mathrm{ml}$ in $5 \mathrm{ml}$ medium. The cells were treated with EA for 15 or $30 \mathrm{~min}$ daily for 14 days. Total RNA from the BMSCs was isolated
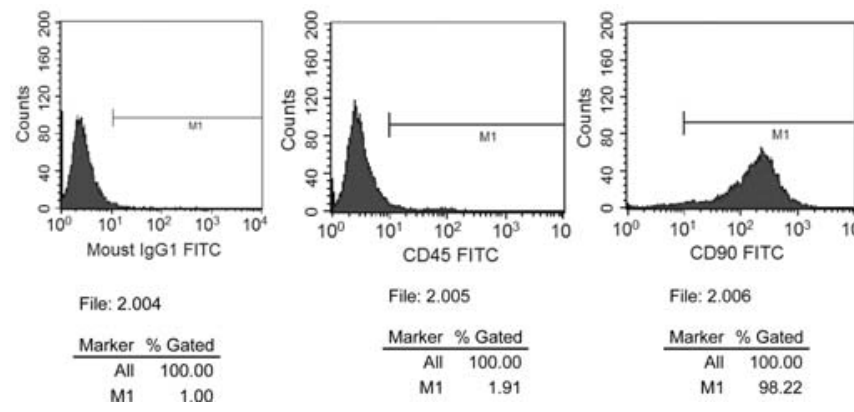

Figure 2. Detection of surface antigens CD45 and CD90 of P3 BMSCs. P3 BMSCs were incubated with saturated CD45-FITC and CD90-FITC monoclonal antibodies at room temperature in the dark for $25 \mathrm{~min}$ and were analyzed by flow cytometry (FCM). Mouse IgG-FITC was used as the isotype control.

with Trizol reagent (Invitrogen). Oligo(dT)-primed RNA $(1 \mu \mathrm{g})$ was reverse-transcribed with SuperScript II reverse transcriptase (Promega) according to the manufacturer's instructions. The obtained cDNA was used to determine the mRNA amount of Sox 9 by PCR. B-actin was used as an internal control. The primers used for amplification of Sox 9 and 3 -actin transcripts are as follows: Sox9 forward 5'-AGC CCT GGT TTC GTT CT-3' and reverse 5'-CTG CTC GTC GGT CAT CTT-3'; ß-actin forward 5'-ACT GGC ATT GTG ATG GAC TC-3' and reverse 5'-CAG CAC TGT GTT GGC ATA GA-3'. The DNA bands were examined using a Gel Documentation system.

Statistical analysis. All data represent the means of three determinations, and data were analyzed using the SPSS package for Windows (version 13.0). Statistical analysis of the data was carried out with the Student's t-test and ANOVA. Differences with $\mathrm{P}<0.05$ were considered statistically significant.

\section{Results}

Expression of BMSC surface antigens. It has been documented that BMSCs are characterized by several phenotypes, including antigens such as CD90, but not by other hemopoietic surface markers, such as CD45 (4). Thus, the purity of BMSCs can be preliminarily measured by positive staining of CD90 and CD45 (5). As shown in Fig. 2, the positive rates of CD90 and CD45 in P3 BMSCs were 98.22 and $1.91 \%$, respectively, indicating the high purity of BMSCs, which provided the experimental basis to investigate the effect of EA on the differentiation of BMSCs into chondrocytes.

Effects of EA on cell morphology. To study the effects of EA on changes in cell morphology, daily observation using optical microscopy was carried out. Fig. 3A and D show that on day 7 or 14 after the routine culture, most P3 BMSCs exhibited a long spindle shape. After EA treatment, the shape of the cells shifted from a spindle to a polygon. As shown in Fig. 3B, C, E and F, most P3 BMSCs exhibited a flat polygon shape, mixed with some protuberant or spindle cells. In addition, we observed the ultrastructural changes in the cells treated with EA for 14 days by TEM. As shown in Fig. 4A 


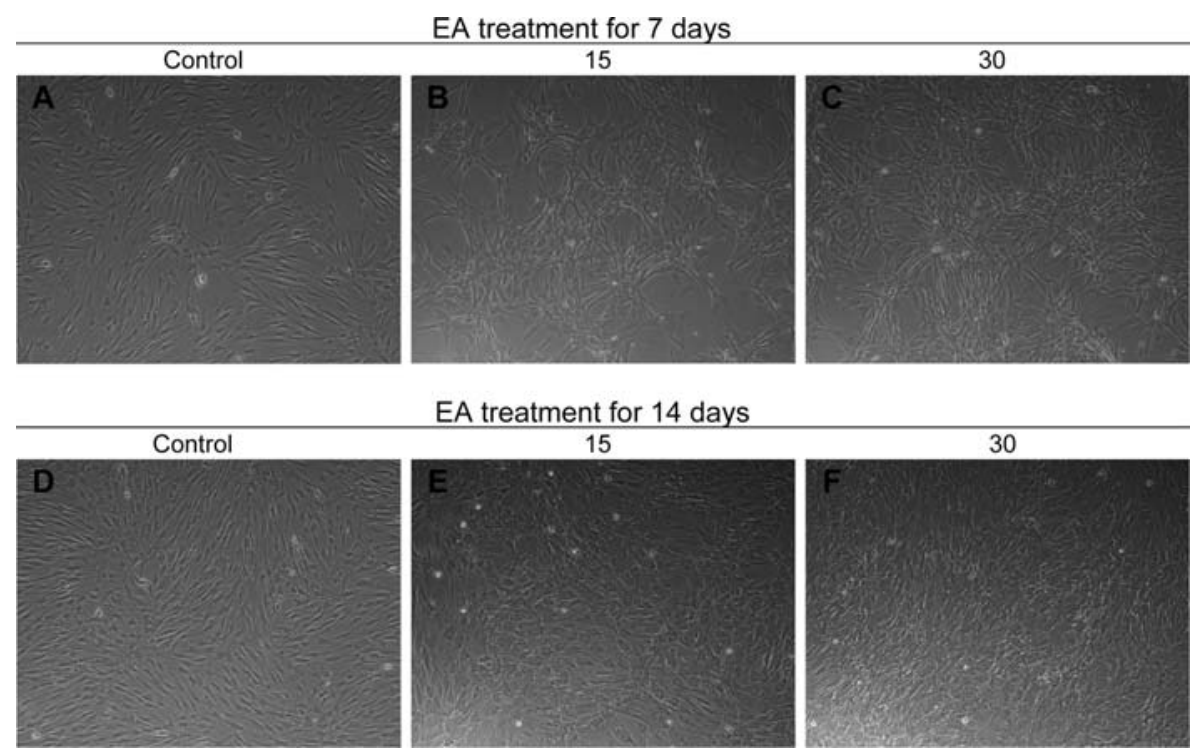

Figure 3. Effect of EA treatment on the morphological changes of P3 BMSCs. P3 BMSCs were treated with EA for 15 (B and E) or 30 (C and F) min daily for 7 (upper panel) or 14 (lower panel) days, and morphological changes were observed using phase-contrast microscopy. The images were captured at a magnification of $\mathrm{x} 100$. Images are representative of three independent experiments.
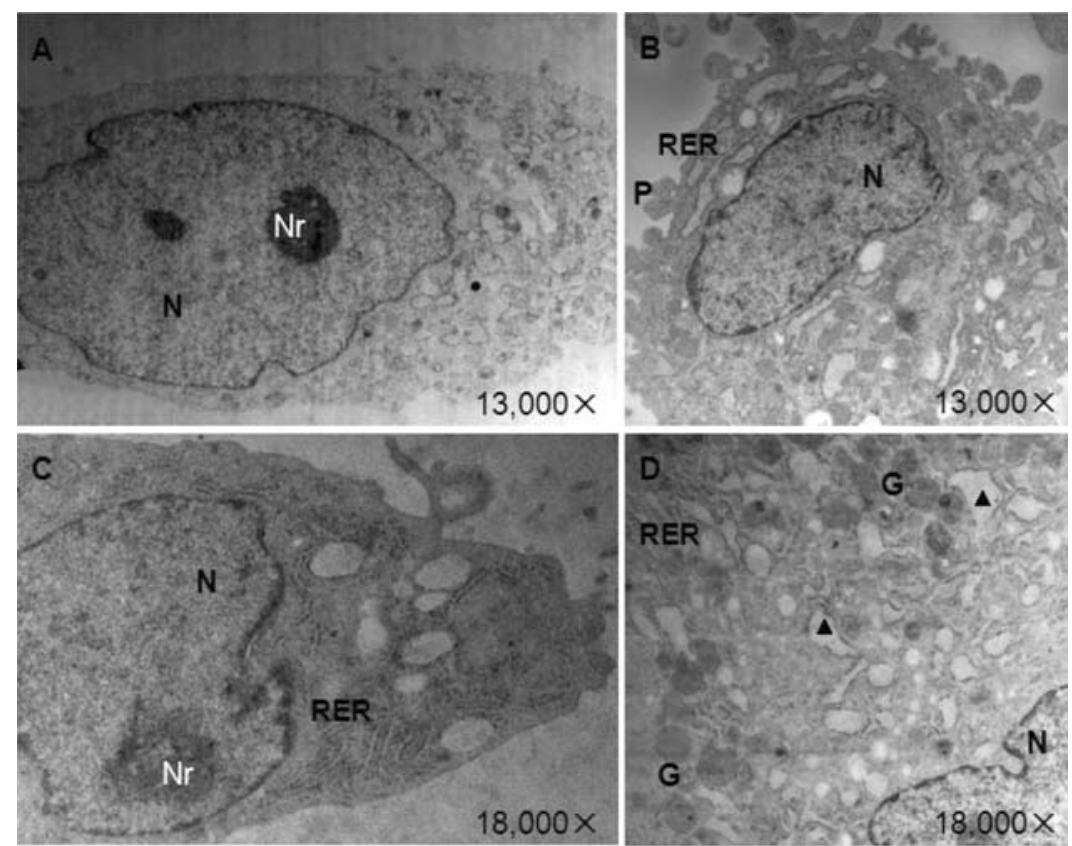

Figure 4. Effect of EA treatment on the ultrastructures of P3 BMSCs. P3 BMSCs were treated with EA for 30 min daily for 14 days (C and D), and ultrastructural changes, compared to the control cells (A and B), were observed using transmission electron microscopy. Images were captured at a magnification of $x 13,000$ or $\times 18,000$. Images are representative of three independent experiments. $\mathrm{N}$, nuclei; $\mathrm{Nr}$, nucleoli; RER, rough endoplasmic reticula; $\mathrm{G}$, secretory granules; P, processes; arrowheads, cisternae.

and $\mathrm{B}$, control cells exhibited a long spindle shape, with a reduced cell content. The cells contained less cytoplasm and fewer organelles, consisting mainly of mitochondria and endoplasmic reticulum. The karyoplasms were comparatively larger, and the nuclei exhibited a near-oval shape. Euchromatin was frequently visible in the nuclear matrices while heterochromatin was less visible. The nucleoli present were larger and nuclear-deviated. As shown in Fig. 4C and D, most EA-treated cells exhibited a near oval shape with more variable cell processes. The cells contained more cytoplasm, in which smooth endoplasmic reticula were abundant and mature, with markedly dilated cisternae and more glycogen particles and a few secretory vesicles; mitochondria and lysosomes were also visible. The nuclear-cytoplasmic ratio decreased, and the nuclei exhibited a moderate or a smaller size, mainly near-oval in shape and occasionally band-like or irregular in shape. Euchromatin was frequently visible in the nuclear matrices, and heterochromatin was mainly located adjacent to the nuclear membrane, with larger nucleoli in some nuclei. These data together suggest that EA induces morphological changes in BMSCs, displaying typical morphology of early chondrocytes. 


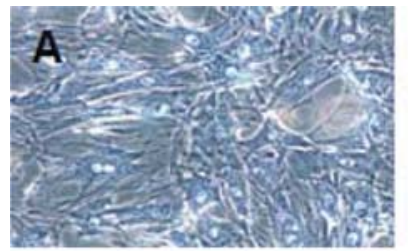

Blank control

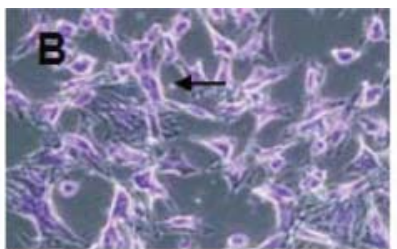

EA 15

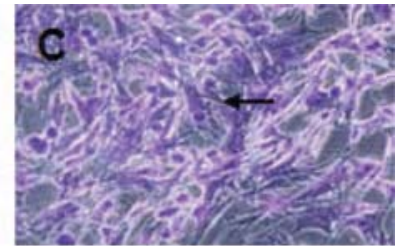

EA 30

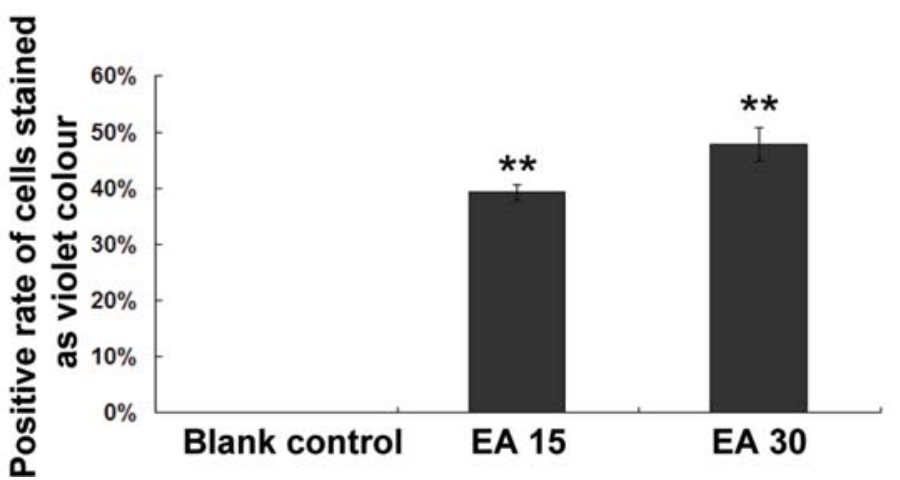

Figure 5. Effect of EA treatment on glycosaminoglycan expression in P3 BMSCs. P3 BMSCs were treated with EA for 15 (B) or 30 (C) min daily for 14 days, and expression of glycosaminoglycan was observed using toluidine blue staining. Images were captured at a magnification of x100, and the positivity was analyzed using the Motic Med 6.0 Digital Medical imaging analysis system. Data represent the averages and SD (error bars) of at least three independent experiments. ${ }^{* *} \mathrm{P}<0.01$, versus control cells. Arrows indicate metachromatically stained cells.

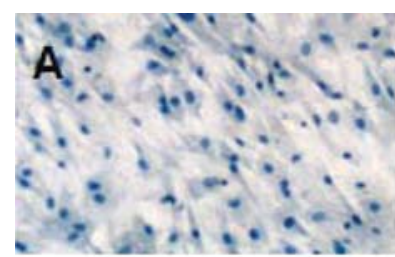

Blank control

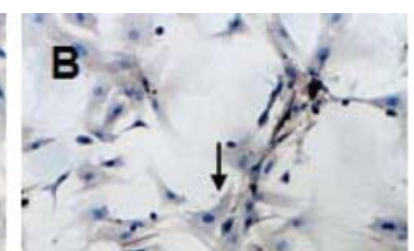

EA 15

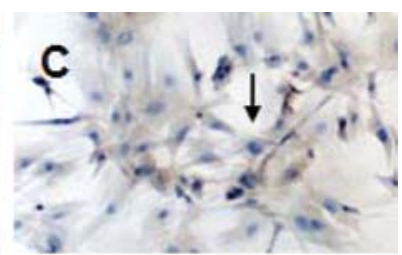

EA 30

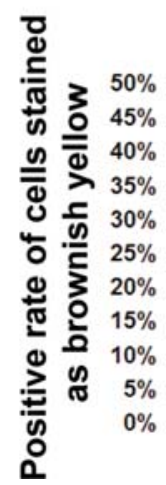

Figure 6. Effect of EA treatment on type II collagen expression of P3 BMSCs. P3 BMSCs were treated with EA for 15 (B) or 30 (C) min daily for 14 days, and expression of type II collagen was observed using immunocytochemical staining. Images were captured at a magnification of x100, and the positivity was analyzed using the Motic Med 6.0 Digital Medical imaging analysis system. The positive rates represent the averages and SD (error bars) of at least three independent experiments. ${ }^{* *} \mathrm{P}<0.01$, versus control cells. Arrows indicate positive cells.

Effects of EA on glycosaminoglycan expression. Glycosaminoglycan exists in the cytoplasm and extracellular matrices of chondrocytes, but not in BMSCs $(18,19)$. Toluidine blue stain is a heterophilic dye, and polysaccharides including glycosaminoglycan can be stained by toluidine blue. To determine the effect of EA on the differentiation of BMSCs into chondrocytes, we examined the expression of glycosaminoglycan by toluidine blue staining. As shown in Fig. 5, the cytoplasm and extra-cellular matrices of the control cells were not positively stained by toluidine blue. In contrast, those of the cells treated with EA for 15 or $30 \mathrm{~min}$ were stained, with a positive rate of $39.31 \pm 1.37$ or $47.96 \pm 2.99 \%$, respectively $(\mathrm{P}<0.01$, versus control cells), indicating that EA significantly and dosedependently induced the differentiation of BMSCs into chondrocytes. 


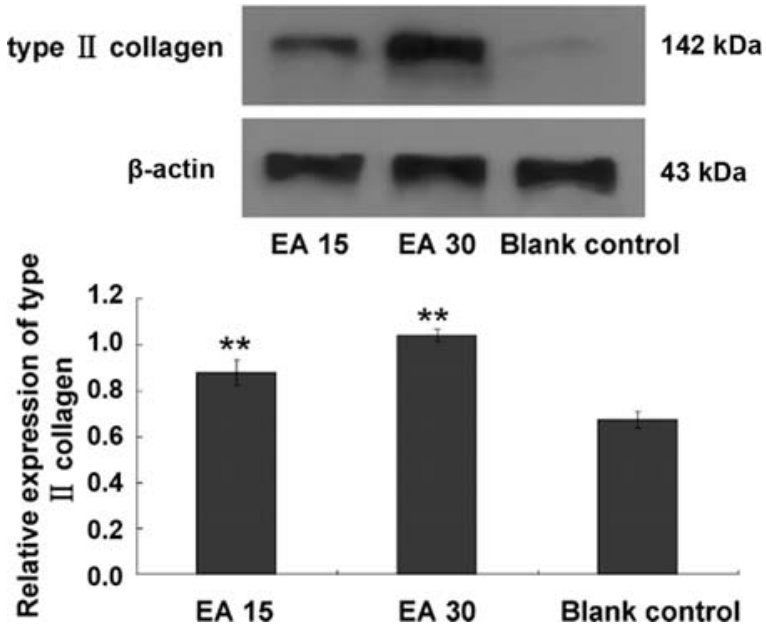

Figure 7. Effect of EA treatment on the expression of type II collagen in P3 BMSCs. P3 BMSCs were treated with EA for 15 or 30 min daily for 14 days, and the protein expression level of type II collagen was analyzed by Western blotting. B-actin was used as the internal controls. Data represent the averages and SD (error bars) from at least three independent experiments. ${ }^{* *} \mathrm{P}<0.01$, versus control cells

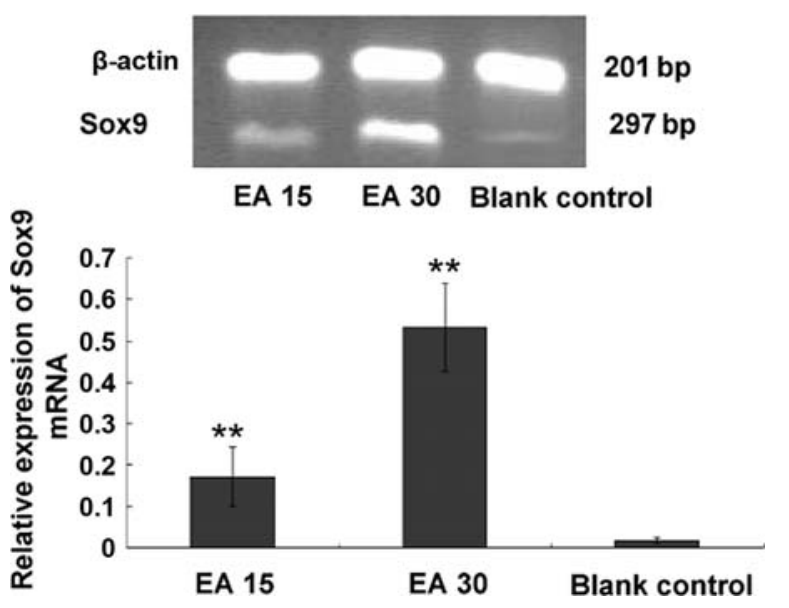

Figure 8. Effect of EA treatment on the expression of Sox9 in P3 BMSCs. P3 BMSCs were treated with EA for 15 or 30 min daily for 14 days, and the mRNA level of Sox 9 was determined by RT-PCR. B-actin was used as the internal control. Data represent the averages and SD (error bars) from at least three independent experiments. ${ }^{* *} \mathrm{P}<0.01$, versus the control cells.

Effects of EA on type II collagen expression. Type II collagen matrix is a chondrocyte-specific protein. Thus, it is a biomarker to distinguish chondrocytes from BMSCs $(20,21)$. To further determine whether EA induces the differentiation of BMSCs into chondrocytes, we evaluated the expression of type II collagen by immunocytochemical staining and Western blotting. Immunocytochemical staining demonstrated that the cytoplasm of cells treated with EA for 15 or 30 min was stained brownish yellow, with a positive rate of $33.23 \pm 2.28$ or $44.32 \pm 2.75 \%$, respectively, whereas the cytoplasm of the control cells was unstained (Fig. 6). Moreover, Western blotting showed that the EA treatment significantly and dosedependently induced expression of the chondrocyte-specific matrix protein type II collagen $(\mathrm{P}<0.01)$ (Fig. 7). These data together confirm that EA induces differentiation of BMSCs into chondrocytes.

Effects of EA on Sox9 mRNA expression. Sox 9 has been reported to be a critical transcription factor involved in the regulation of chondrogenesis. During chondrogenesis in the mouse, Sox 9 is co-expressed with Col2a1, the gene encoding type 11 collagen, the major cartilage matrix protein $(22,23)$ which demonstrates that Col2a1 expression is directly regulated to Sox 9 protein in vivo and implicates abnormal regulation of Col2a1 during chondrogenesis as a cause of skeletal abnormalities associated with campomelic dysplasia. To investigate the mechanism by which EA treatment regulates the expression of type II collagen, we examined Sox 9 mRNA by RT-PCR. As shown in Fig. 8, EA treatment significantly and dose-dependently promoted the expression of Sox 9 mRNA $(\mathrm{P}<0.01)$, suggesting that EA-induced expression of type II collagen is mediated through the effective up-regulation of Sox9.

\section{Discussion}

BMSCs have been established as seed cells and gene vectors available for the replacement therapy of multiple disorders, and their in vitro isolation and culture is already well developed (24-27). However, development of methods able to induce the committed differentiation of stem cells, has become the focus of tissue engineering development. Thus, an effective and safe modality of induction is valuable since potential safety concerns have arisen from the previous differentiation of BMSCs induced by agents $(9,10)$.

In this study, we demonstrated that EA treatment can successfully induce BMSCs into chondrocytes. After EA treatment, the cell morphology shifted significantly from a spindle to a polygon shape. TEM revealed that the rough endoplasmic reticula and other organelles were more abundant and better developed in the EA-treated cells than in the untreated cells, with markedly dilated endoplasmic reticula, indicating enhanced cell metabolism. Nuclear morphology was mostly near-oval, with occasional band-like or irregular shapes, suggesting the differentiation of BMSCs into various cell phenotypes consistent with the multipotent differentiation of BMSCs. In the present study, some induced cells did not present typical structures of mature chondrocytes. This could have been due to some cells remaining in an undifferentiated state, due to inadequate treatment by EA. The mechanism involved needs further investigation.

In addition, we found that the cytoplasm and extracellular matrices were metachromatically stained by toluidine blue in the treated cells in a dose-dependent manner, and EA treatment dose-dependently and significantly induced the expression of type II collagen protein. These morphological findings justified the differentiation of BMSCs into chondrocytes by EA and suggest that EA treatment induces secretion by BMSCs of chondrocyte-specific matrices of glycosaminoglycan and type II collagen. Western blotting further confirmed that EA treatment promoted the protein expression of type II collagen in a dose-dependent manner. Additionally, the non-induced cells also expressed minimal 
type II collagen, possibly from spontaneous chondrocyte differentiation at some locations with higher cell density in the culture (28).

Many transcription factors participate in the course of the differentiation of BMSCs into chondrocytes. Sox9 is one of the most important factors, necessary in the regulation of BMSCs into progenitor cells and aggregation of MSCs. This process is not only essential for the expression of type II collagen (29), but also regulates the expression of cell membrane protein which is vital in the process of the aggregation of MSCs. Moreover, Sox9 plays a role in the regulation of the further differentiation of chondrocytes, multiplicational chondrocytes into hypertrophic chondrocytes, which maintains the proliferation of chondrocytes and inhibits their differentiation into hypertrophic chondrocytes (30). Therefore, in the present study, Sox9 was used as one of the indices to measure the effects of EA on the differentiation of BMSCs into chondrocytes. The RT-PCR assay of Sox 9 mRNA showed that EA treatment promoted the expression of Sox 9 mRNA in a dose-dependent manner, which was found to be generally synchronized with the EA frequency shift cycle and respiratory rhythm and associated with the window effects of an electrical field on cell behavior (31). The concrete mechanisms of action remain unknown, as they have not yet been investigated. The expression of Sox9 mRNA increased with time in a statistically significant manner $(\mathrm{P}<0.05)$, indicating the potential cumulative effects of stimulatory EA on cells through an unknown mechanism. Based on the above results, we conclude that the expression of type II collagen was mediated through the promotion of Sox9, consistent with a previous study (23). However, it is still unknown whether EA treatment induces the differentiation of BMSCs into late stage chondrocytes. Transcription factor Runx 2 is expressed in the late stage of chondrogenesis, mainly expressed during chondrocyte hypertrophy, which promotes collage $\mathrm{X}$ expression, a hypertrophic chondrocyte marker. Therefore, expression of Runx 2 and type X collagen will be investigated by us in a subsequent study.

In summary, daily interference with low-frequency EA of $2 \mathrm{~Hz}$ with square-wave burst pulses (duration $1.1 \mathrm{sec}$ ) and a burst frequency of $100 \mathrm{~Hz}$ (duration $2.2 \mathrm{sec}$ ) with alternating polarity significantly and dose-dependently induced the differentiation of BMSCs into early stage chondrocytes in vitro and the secretion of glycosaminoglycan and type II collagen, which was mediated by the transcription factor Sox 9 .

\section{Acknowledgements}

This research was supported by 2008 Fujian Province Small Creations and Projects (no.1) and Key Project of Traditional Chinese Medicine of Fujian Provincial Health Department (WZZG0601).

\section{References}

1. Cohen I, Robinson D, Melamed E and Nevo Z: Use of a novel joint-simulating culture system to grow organized ex-vivo threedimensional cartilage-like constructs from embryonic epiphyseal cells. Iowa Orthop J 25: 102-107, 2005.

2. Gregory CA, Prockop DJ and Spees JL: Non-hematopoietic bone marrow stem cells: molecular control of expansion and differentiation. Exp Cell Res 306: 330-335, 2005.
3. Phinney DG and Prockop DJ: Concise review: mesenchymal stem/multipotent stromal cells: the state of transdifferentiation and modes of tissue repair-current views. Stem Cells 25: 2896-2902, 2007.

4. Wexler SA, Donaldson C, Denning-Kendall P, Rice C, Bradley B and Hows JM: Adult bone marrow is a rich source of human mesenchymal 'stem' cells but umbilical cord and mobilized adult blood are not. Br J Haematol 121: 368-374, 2003.

5. Wagner W, Wein F, Seckinger A, Frankhauser M, Wirkner U, Krause U, Blake J, Schwager C, Eckstein V, Ansorge W and Ho AD: Comparative characteristics of mesenchymal stem cells from human bone marrow, adipose tissue, and umbilical cord blood. Exp Hematol 33: 1402-1416, 2005.

6. Ng F, Boucher S, Koh S, Sastry Konduru SR, Chase L, Lakshmipathy U, Choong C, Yang Z, Vemuri MC, Rao MS and Tanavde V: PDGF, TGF- $\beta$, and FGF signaling is important for differentiation and growth of mesenchymal stem cells (MSCs): transcriptional profiling can identify markers and signaling pathways important in differentiation of MSCs into adipogenic, chondrogenic, and osteogenic lineages. Blood 112: 295-307, 2008.

7. Deng J, Jin H, Liu J and Liu Y: The differentiation potential of rat bone marrow stem cells to cartilage and integrin antibody can blocking this course in vitro. FASEB J 23: 1030-1037, 2009.

8. Kaptchuk TJ: Acupuncture: theory, efficacy, and practice. Ann Intern Med 136: 374-383, 2002.

9. NIH Concensus Conference: Acupuncture. JAMA 280: 1518-1524, 1998.

10. Jubb RW, Tukmachi ES, Jones PW, Dempsey E, Waterhouse L and Brailsford S: A blinded randomised trial of acupuncture (manual and electroacupuncture) compared with a non-penetrating sham for the symptoms of osteoarthritis of the knee. Acupunct Med 26: 69-78, 2008.

11. Tukmachi E, Jubb R, Dempsey E and Jones P: The effect of acupuncture on the symptoms of knee osteoarthritis - an open randomised controlled study. Acupunct Med 22: 14, 2004.

12. Vas J and White A: Evidence from RCTs on optimal acupuncture treatment for knee osteoarthritis - an exploratory review. Acupunct Med 25: 29-35, 2007.

13. Aoki E, Kasahara T, Hagiwara H, Sunaga M, Hisamitsu N and Hisamitsu T: Electroacupuncture and moxibustion influence the lipopolysaccharide-induced $\mathrm{TNF}-\alpha$ production by macrophages In Vivo 19: 495-500, 2005.

14. Takaoka Y, Ohta M, Ito A, Takamatsu K, Sugano A, Funakoshi K, Takaoka N, Sato N, Yokozaki H, Arizono N, Goto S and Maeda E: Electroacupuncture suppresses myostatin gene expression: cell proliferative reaction in mouse skeletal muscle. Physiol Genomics 30: 102-110, 2007.

15. Zhang RX, Li A, Liu B, Wang L, Ren K, Qiao JT, Brian MB and Lao L: Electroacupuncture attenuates bone cancer pain and inhibits spinal interleukin-1ß expression in a rat model. Anesth Analg 105: 1482-1488, 2007.

16. Sun Z, Li X, Su Z, Zhao Y, Zhang L and Wu M: Electroacupuncture enhanced differentiation of bone marrow stromal cells into neuronal cells. J Sport Rehabil 18: 398-406, 2009.

17. The Ministry of Science and Technology of the People's Republic of China: Guidance Suggestions for the Care and Use of Laboratory Animals, 2006.

18. Struglics A, Larsson S, Pratta MA, Kumar S, Lark MW and Lohmander LS: Human osteoarthritis synovial fluid and joint cartilage contain both aggrecanase- and matrix metalloproteinase-generated aggrecan fragments. Osteoarthritis Cartilage 14: 101-113, 2006.

19. Nakaoka R, Hsiong SX and Mooney DJ: Regulation of chondrocyte differentiation level via co-culture with osteoblasts. Tissue Eng 12: 2425-2433, 2006.

20. Grimmer C, Balbus N, Lang U, Aigner T, Cramer T, Muller L, Swoboda B and Pfander D: Regulation of type II collagen synthesis during osteoarthritis by prolyl-4-hydroxylases: possible influence of low oxygen levels. Am J Pathol 169: 491-502, 2006.

21. Tsuji K, Bandyopadhyay A, Harfe BD, Cox K, Kakar S, Gerstenfeld L, Einhorn T, Tabin CJ and Rosen V: BMP2 activity, although dispensable for bone formation, is required for the initiation of fracture healing. Nat Genet 38: 1424-1429, 2006.

22. Ng LJ, Wheatley SC, Muscat GE, Conway-Campbell J, Bowles J, Wright E, Bell DM, Tam PP, Cheah KS and Koopman P: SOX9 binds DNA, activates transcription, and coexpresses with type II collagen during chondrogenesis in the mouse. Dev Biol 183: 108-121, 1997. 
23. Bell DM, Leung KK, Wheatley SC, Ng LJ, Zhou S, Ling KW, Sham MH, Koopman P, Tam PP and Cheah KS: SOX9 directly regulates the type-II collagen gene. Nat Genet 16: 174-178, 1997.

24. Zhang X, Tang T, Shi Q, Fernandes JC and Dai K: The immunologic properties of undifferentiated and osteogenic differentiated mouse mesenchymal stem cells and its potential application in bone regeneration. Immunobiology 214: 179-186, 2009.

25. Miljkovic ND, Cooper GM and Marra KG: Chondrogenesis, bone morphogenetic protein-4 and mesenchymal stem cells Osteoarthritis Cartilage 16: 1121-1130,2008.

26. Kuo CK and Tuan RS: Tissue engineering with mesenchymal stem cells. IEEE Eng Med Biol Mag 22: 51-56, 2003.

27. Shin M, Yoshimoto H and Vacanti JP: In vivo bone tissue engineering using mesenchymal stem cells on a novel electrospun nanofibrous scaffold. Tissue Eng 10: 33-41, 2004.
28. Schmitt B, Ringe J, Haupl T, Notter M, Manz R, Burmester GR, Sittinger M and Kaps C: BMP2 initiates chondrogenic lineage development of adult human mesenchymal stem cells in highdensity culture. Differentiation 71: 567-577, 2003.

29. Bi W, Deng JM, Zhang Z, Behringer RR and de Crombrugghe B: Sox9 is required for cartilage formation. Nat Genet 22: 85-89, 1999.

30. Akiyama H, Chaboissier MC, Martin JF, Schedl A and de Crombrugghe B: The transcription factor Sox9 has essential roles in successive steps of the chondrocyte differentiation pathway and is required for expression of Sox 5 and Sox6. Genes Dev 16: 2813-2828, 2002.

31. Binhi VN and Goldman RJ: Ion-protein dissociation predicts 'windows' in electric field-induced wound-cell proliferation. Biochim Biophys Acta 1474: 147-156, 2000. 